Research Paper

\title{
Identification of GINS2 prognostic potential and involvement in immune cell infiltration in hepatocellular carcinoma
}

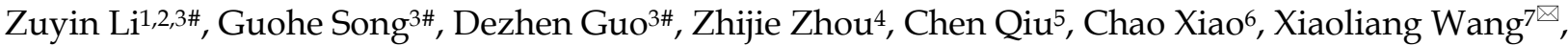 \\ Yupeng Wang ${ }^{3 凶}$
}

1. Department of Hepatobiliary Surgery, Peking University Organ Transplantation Institute, Peking University People's Hospital, Beijing, 100034, China.

2. Department of General Surgery, Shanghai General Hospital, Shanghai Jiao Tong University School of Medicine, Shanghai, 201600, China.

3. Department of Liver Surgery and Transplantation, Liver Cancer Institute, Zhongshan Hospital, Fudan University; Key Laboratory of Carcinogenesis and Cancer Invasion (Ministry of Education), Fudan University, Shanghai, 200032, China.

4. Department of General Surgery, Huashan Hospital North, Fudan University, Shanghai 201907, China.

5. Institute of Gallstone Disease, Center of Gallbladder Disease, Shanghai East Hospital, Tongji University School of Medicine, Shanghai, 200120, China.

6. Department of General Surgery, Huashan Hospital, Fudan University, Shanghai, 20040, China.

7. Department of General Surgery, Qingpu Branch of Zhongshan Hospital Affiliated to Fudan University, Shanghai, 201700, China.

\#Zuyin Li, Guohe Song and Dezhen Guo contributed equally to the work.

$\triangle$ Corresponding authors: Yupeng Wang, Department of General Surgery, Shanghai General Hospital, Shanghai Jiao Tong University School of Medicine, Shanghai, 201600, China; Department of Liver Surgery and Transplantation, Liver Cancer Institute, Zhongshan Hospital, Fudan University; Key Laboratory of Carcinogenesis and Cancer Invasion (Ministry of Education), Fudan University, Shanghai 200032, China. E-mail: wypyiyun08@sina.com; Xiaoliang Wang, Department of General Surgery, Qingpu Branch of Zhongshan Hospital Affiliated to Fudan University. 1158 Park Road(E), Qingpu Shanghai, 201700, China.

E-mail: xiaoliangwang1975@hotmail.com

(c) The author(s). This is an open access article distributed under the terms of the Creative Commons Attribution License (https://creativecommons.org/licenses/by/4.0/). See http://ivyspring.com/terms for full terms and conditions.

Received: 2021.08.03; Accepted: 2021.12.11; Published: 2022.01.01

\begin{abstract}
Background: GINS2 has been reported to have prognostic value in several solid tumors other than hepatocellular carcinoma $(\mathrm{HCC})$, and its influence on tumor immunity has not been investigated thus far.

Methods: The transcriptome profiles were retrieved from two public databases, GEO and TCGA. The median GINS2 expression was considered as cutoff to define GINS2 high and GINS2low groups and to obtain differentially expressed genes. These genes were then subjected to KEGG pathway and gene ontology (GO) analysis and to gene set enrichment analysis (GSEA). Survival analyses according to GINS2 level were performed utilizing Kaplan-Meier plotter. TIMER database was adopted to investigate associations between GINS2 level and infiltrating immunocytes, and the correlation between immunocyte-related gene expression and GINS2 level was evaluated via GEPIA database. A 236-patient validation cohort were applied to confirm the bioinformatic results of TCGA and TIMER database.

Results: GINS2 is augmented in tumorous tissues of HCC patients compared with nontumor specimens, and GINS2-overexpressed patients have poorer overall survival (OS) and disease-specific survival (DSS) than those with low GINS2 expression in HCC ( $P=0.009$ and $P=0.002$ respectively). Cell cycle and DNA replication were two main processes that enriched in tumor cells overexpressed GINS2 gene (NES = 1.848, $P=0.007$; and NES $=1.907, P=0.005$, respectively). Moreover, GINS2 correlates positively with markers of activated CD8 ${ }^{+}$ and $\mathrm{CD} 4{ }^{+} \mathrm{T}$ cells, as well as exhausted $\mathrm{T}$ lymphocytes.

Conclusions: HCC patients overexpressed GINS2 have poorer prognoses than those with low GINS2 expression, possibly as a result of the function of GINS2 in cell cycle and DNA replication as well the exhaustion of T lymphocytes.
\end{abstract}

Key words: GINS2, DNA replication, cell cycle, immune cell infiltration.

\section{Introduction}

Hepatocellular carcinoma is currently the sixth of top ten incidence of malignant tumor among both males and females worldwide but the fourth deadly disease of cancer [1]. Accumulating evidence reveal 
that the biological behavior of cancer cells is tightly related with diverse elements of tumor microenvironment (TME), say, immune cells. They function as key factors in TME and are of great significance in tumorigenesis and progression [2]. With the gradual understanding of TME in HCC, the appropriate stratification in patients may gain great benefit from targeted immunotherapies [3].

When tumor cells are growing and prosper, they seek to compromise immune surveillance within the microenvironment [4]. The molecules, say, PD-1/PD-L1 interaction, act as a key role in this process [5]. Researchers noticed that the levels of PD-1 in $\mathrm{CD}^{+} \mathrm{T}$ cells was elevated in patients with liver cancer, and analyses of samples from patients who have not obtained any medical treatment show the infiltrating abundance of PD- $1^{+} \mathrm{CD} 8^{+} \mathrm{T}$ cells is related with poor outcomes [6-8]. Moreover, it has also been discovered in HCC patients that the overexpressed PD-L1 on cancerous cells can lead to T cell exhaustion and functional incompetence [9]. Hence, the goal of immunotherapy is to activate the patient's own incompetent or suppressed immune system to generate antitumor effects. Increasing evidence has demonstrated that dysregulated gene expression is correlated with immune abnormalities, as well as dysfunction of multiple biological processes, including dysregulated cell cycle and DNA replication processes, although the complex regulatory network involving these genes is still unknown. Therefore, in-depth analysis of these genes will help understand the mechanistic basis for tumorigenesis and development of HCC and clarify the interaction between the molecules and immune infiltration.

GINS complex subunit 2 (GINS2), systematically identified in 2003, is an essential protein for GINS, a pivotal complex for the processes of DNA replication [10]. GINS2 can bind preferentially to single-stranded DNA, which is especially significant to ensure the orderly progress of DNA replication in many cell types and organs [11]. In 2009, GINS2 was first suggested as a crucial gene promoting metastasis in breast cancer [12]. Previous studies indicate that downregulation of GINS2 causes decreased cell vitality, promotes apoptosis and the inhibition cell cycle progression in pancreatic tumor cells [13]. These evidence hints that GINS2 functions as a pivotal molecule in tumorigenesis. In addition, some reports have revealed that GINS2 overexpression bring unfavorable outcomes in different tumor types, such as non-small-cell lung cancer (NSCLC), breast and cervical cancer [14-16]. However, there are few reports on the utility of GINS2 as a biomarker for the prognosis of HCC and its involvement in immune infiltration. Moreover, the underlying functional and mechanistic basis of GINS2 in tumor proliferation and TME remain unknown.

In the present study, we identified GINS2 overexpression in HCC patients and its impact on the clinical outcomes of HCC patients and explored the association of GINS2 with immunocyte infiltration as well as immune marker gene sets via TIMER and GEPIA database as well as a 236-patient validation cohort. Our results demonstrated the significance of GINS2 in the occurrence and progression of hepatocellular carcinoma and illustrated the association between the levels of GINS2 and prognosis as well as immune cell infiltration.

\section{Materials and methods}

\section{Data resources and descriptions}

Five RNA-sequencing datasets (The sample size exceeds 100 cases), GSE77314, GSE45436, GSE36376, GSE25097 and GSE14520, including HCC tumorous and nontumor specimens were retrieved from Gene Expression Omnibus (GEO, https://www.ncbi. nlm.nih.gov/geo/). The details of each GEO datasets are presented in Table S1. The raw count data and clinical information of TCGA-LIHC cohort were obtained from Genomic Data Commons Data Portal (https://cancergenome.nih.gov/). The RNA-seq dataset contains 421 samples, including 371 tumorous tissues and 50 normal liver specimens. The "edgeR" package is applied to analyze the levels of GINS2 between tumorous and nontumor specimens.

\section{Patients and tissue samples}

To prove the results from database, we collected 236 patients with HCC to form a validation cohort. This cohort consisted of 236 patients who were diagnosed with HCC in the Department of Liver Surgery and Transplantation, Zhongshan Hospital, Fudan University, between 2007 and 2008. Clinical information was summarized from electronic medical records. Tissue samples collected during surgery were pathologically examined, fixed in $4 \%$ paraformaldehyde and stored at the tissue bank before analysis. The study was approved by the Ethical Committees of Zhongshan Hospital, and each subject was fully informed and signed the written consent.

\section{Immunohistochemical (IHC) staining and evaluation of GINS2 protein expression}

Sections of HCC samples and adjacent normal specimens were incubated with a rabbit polyclonal anti-GINS2 antibody (1:200, \#ab197123, Abcam, Cambridge, UK). The results of IHC staining were examined under double-blinded conditions and 
scored on a semi-quantitative scale based on the score of intensity and extent. The score of intensity was marked as 0 (no staining), 1 (weak), 2 (moderate), and 3 (strong), and the percentage of positive stained area as 0 (none), $1(1-25 \%), 2$ (26-50\%), 3 (51-75\%), and 4 $(>=75 \%)$. The IHC score was calculated by multiplying the score of intensity and extent in each sample (scale range 0-12). The levels of GINS2 were divided into "GINS2 Low" group (score <6) and "GINS2 High" group (scores >=6).

\section{Multiplex immunohistochemistry (mIHC)}

TSA plus fluorescent multiple staining kit (\#G1236-100T, Servicebio) was applied for multiplex IHC. The 5-mm formalin-fixed and parrffin-embedded slides were deparaffinized and rehydrated. All slides were subjected to epitope retrieval with EDTA ( $\mathrm{pH} \mathrm{8.0)}$ for $8 \mathrm{~min}$. After cooling $\left(25^{\circ} \mathrm{C}\right)$, slides were washed with PBS $(3 * 5 \mathrm{~min})$, and endogenous peroxidase activity were blocked with $\mathrm{H}_{2} \mathrm{O}_{2}$ (3\%) for $25 \mathrm{~min}$. Then blocking buffer (5\% BSA, Solarbio, \#SW3015) was used for 30 min-protein blocking. All slides were incubated with antibody against CD8 (\#GB13068, Servicebio, 1:500) at $4^{\circ} \mathrm{C}$ overnight. After washes, sections were incubated with an HRP-conjugated secondary antibody for $50 \mathrm{~min}$. Then slides were dyed with CY3-TSA for $10 \mathrm{~min}$. This method was applied three more times using the antibodies as follows, GINS2 (Proteintech, \#16247-1-AP, 1:1000, dye FITC), CD4 (Wisee Biotechnology, \#YX32005-100, 1:1000, dye 647-TSA), and CD3 (Servicebio, \#GB13440, 1:100, dye594). EDTA ( $\mathrm{pH}$ 8.0) buffer was applied for the next round of epitope retrieval with a cooker $\left(125^{\circ} \mathrm{C}, 15 \mathrm{~min}\right)$. Cell nucleus was labeled with DAPI (Servicebio, \#G1012) and covered with Antifade Mounting Medium (Beyotime, \#P0126). Secondary antibodies were used as follows: anti-rabbit (1:500, Servicebio, GB23303) and anti-mouse (1:500, Servicebio, GB23301).

\section{Differentially expressed gene (DEGs) analysis}

In the TCGA dataset, we analyzed DEGs by applying the "edgeR" package between GINS2 high and GINS2 ${ }^{\text {low }}$ group defined by the median expression of GINS2. Fold change $>2$ and adjusted $P$ value (Benjamini and Hochberg adjustment) $<0.05$ were used as the cutoffs for DEGs. To determine the functions of the 421 upregulated DEGs, these genes were then subjected to DAVID 6.8 (https://david.ncifcrf.gov/) for KEGG pathway and GO functional analyses and via Cytoscape software for result visualization, as summarized by ClueGo modules. Correlations between GINS2 and cell cycleor DNA replication-related genes were determined by Prism 8 software with Pearson's R and $P$ values.

\section{Kaplan-Meier plotter database analysis}

Kaplan-Meier plotter can evaluate the impact of 54,000 genes (encoding mRNA, miRNA, and protein) on the prognosis of 21 tumor types, including HCC $(n=364)$. The relationship between the levels of GINS2 and survival in HCC was evaluated via the liver cancer module (http://kmplot.com/analysis/)[17]. Hazard ratio (HR), 95\% confidence interval (CI), and log-rank P-value were presented. For subgroup analysis, we chose all default settings and applied the survival analysis.

\section{Immune infiltration analysis}

TIMER database provides resources to comprehensively investigate the immune infiltration of all tumor types (https://cistrome.shinyapps. io/timer/) [18]. Analysis of GINS2 expression level was performed in multiple cancer types via the DiffExp module, and the association between the GINS2 levels and infiltrating immunocytes was determined through gene modules. The correlation modules can assess the relation between GINS2 levels and markers of tumor-infiltrating immune cells. We chose the immune-related markers presented in previous studies [19-21]. The correlation module was utilized to draw expression scatterplots, and Spearman's rho value was calculated to estimate statistical significance.

\section{Correlation analysis from GEPIA database}

GEPIA database is a multifunctional web tool that contains 9,736 tumor and 8,587 normal specimens from the TCGA and GTEx database, which analyze RNA-seq profiles [22]. In this study, the tool was applied to further verify the association between GINS2 and marker gene sets of monocytes and Tumor-associated macrophages (TAMs). The Pearson method was adopted to calculate the correlation coefficient. The analysis include tumor and nontumor tissue datasets. The $\mathrm{R}$ and $P$ values for the correlation between GINS2 and genes of interest generated by GEPIA are summarized in Table 4. $P<0.05$ was considered statistically significant.

\section{Gene set enrichment analysis (GSEA)}

To discover potential biological differences among high- and low-GINS2 expression in the liver of HCC patients, we utilized GSEA to obtain relevant pathways using GSEA. As mentioned before, we split all HCC patients into GINS2 high and GINS2 ${ }^{\text {low }}$ groups. The ranks of all genes were obtained through their differences between two groups. GSEA concentrates on the expression data at the levels of specific gene sets. The normalized enrichment score (NES) was calculated. We chose 1000 times in the process of the 
gene set permutations and set a $P$-value cutoff of 0.05 .

\section{Statistical analysis}

Differences in the transcriptional levels of gene among the defined groups were evaluated via Student's t-test or Mann-Whitney U-test based on variable type. Chi-squared test was utilized to evaluate the relation between GINS2 levels and clinicopathological parameters. Statistical software (SPSS, version 25.0) from SPSS Inc. (Chicago, IL, USA) was used. A two-tailed $P<0.05$ was considered significant for all analyses.

\section{Results}

\section{Upregulated GINS2 in tumor tissues predicts poorer prognoses of HCC patients}

To evaluate the transcriptional levels of GINS2 gene in tumor and nontumor tissues, five GEO expression microarray datasets and expression profiles from the TCGA-LIHC cohort were used. By contrast, GINS2 gene was significantly overexpressed in tumor samples of the GSE77314, GSE45436, GSE36376, GSE25097, GSE14520 and TCGA datasets (all $\mathrm{P}<0.01$, Figure 1A). Moreover, the transcriptional data from the GEPIA database also confirmed the overexpression of GINS2 in tumor samples ( $\mathrm{T}$, red, Figure 1B). To further prove these results in the database, we performed IHC staining of tissues from a 236-patient validation cohort. The IHC analysis showed that the protein expression of GINS2 in tumor samples was significantly higher than those in matched nontumor tissues (Figure 1C). Next, we examined the GINS2 expression in TIMER database, which extracts expression profiles of multiple malignancies from TCGA. GINS2 expression was undoubtedly higher in HCC tumor samples than in normal specimens and showed a pan-cancer overexpression signature (Figure S1). Collectively, these results showed significant overexpression of GINS2 in HCC patients.

To explore the impacts of GINS2 overexpression on HCC prognosis, we determined the correlation between clinicopathological characteristics and GINS2 expression status in the TCGA cohort. We split the TCGA-LIHC cohort equally in two groups. Patients with GINS2 expression higher than the median expression value of GINS2 were deemed as "high" group and the rest, "low" group. The results revealed a tight correlation between GINS2 overexpression and T stage as well as pathologic stage of HCC patients (Table 1 , all $P<0.05$ ). Our validation cohort also confirmed that the overexpression of GINS2 was related with TMN stage (Table S2). Furthermore, we analyzed the levels of GINS2 expression in TCGA cohort to evaluate the role of GINS2 gene in the outcomes of patients. Overall survival (OS, $P=0.009$ ), disease-specific survival (DSS, $P=0.002$ ), progression-free survival (PFS, $P=0.018$ ) and relapse-free survival (RFS, $P=0.010$ ) analysis showed that GINS2 overexpression was generally correlated with shorter survival time and poorer prognosis in HCC patients (Figure 1D). The result of OS $(P=0.009)$ was further verified in the validation cohort (Figure 1E). We additionally applied subgroup survival analysis in different populations. The results revealed that GINS2 overexpression contributed to worse OS and DFS in male HCC cohort, and a high GINS2 level caused unfavorable OS and DSS in patients without a history of alcohol consumption or hepatitis virus infection (Figure S2A and S2B). Moreover, high GINS2 expression was a risk factor for OS and DSS in Asian HCC patients (Figure S2A and S2B). Collectively, the above evidences demonstrate that GINS2 overexpression is significantly associated with unfavorable survival outcomes in HCC patients.

Table 1. Clinicopathological characteristics in relation to GINS2 expression status in TCGA cohort.

\begin{tabular}{|c|c|c|c|c|c|}
\hline \multirow[t]{2}{*}{ Characteristics } & \multirow{2}{*}{$\begin{array}{l}\text { TCGA } \\
\text { Cohort } \\
(\mathrm{N}=371)\end{array}$} & \multicolumn{4}{|c|}{ GINS2 expression } \\
\hline & & $\begin{array}{l}\text { High } \\
N=185(\%)\end{array}$ & $\begin{array}{l}\text { Low } \\
\mathrm{N}=186(\%)\end{array}$ & $x^{2}$ & $P$ value \\
\hline \multicolumn{6}{|l|}{ Age } \\
\hline$>=70 \mathrm{y}$ & & $145(50.3)$ & $143(49.7)$ & 0.120 & 0.729 \\
\hline$<70 y$ & & $40(48.2)$ & $43(51.8)$ & & \\
\hline \multicolumn{6}{|l|}{ Gender } \\
\hline male & & $123(49.2)$ & $127(50.8)$ & 0.136 & 0.713 \\
\hline female & & $62(51.2)$ & $59(48.8)$ & & \\
\hline \multicolumn{6}{|l|}{ Race } \\
\hline ASIAN & & $92(58.2)$ & $66(41.8)$ & 7.836 & 0.098 \\
\hline $\begin{array}{l}\text { BLACK or AFRICAN } \\
\text { AMERICAN }\end{array}$ & & $7(41.2)$ & $10(58.8)$ & & \\
\hline WHITE & & $81(44.0)$ & $103(56.0)$ & & \\
\hline $\begin{array}{l}\text { AMERICAN INDIAN or } \\
\text { ALASKA NATIVE }\end{array}$ & & $1(50)$ & $1(50)$ & & \\
\hline NA & & $4(40)$ & $6(60))$ & & \\
\hline \multicolumn{6}{|l|}{$\mathbf{T}$} \\
\hline T1-T2 & & $126(45.7)$ & $140(54.3)$ & 7.653 & $0.006^{*}$ \\
\hline T3-T4 & & $59(62.1)$ & $36(37.9)$ & & \\
\hline \multicolumn{6}{|l|}{$\mathbf{N}$} \\
\hline No & & $133(52.8)$ & $119(47.2)$ & 4.494 & 0.213 \\
\hline N1 & & $1(25)$ & $3(75)$ & & \\
\hline NX & & $50(43.9)$ & $64(56.1)$ & & \\
\hline NA & & $1(100)$ & $0(0)$ & & \\
\hline \multicolumn{6}{|l|}{$\mathbf{M}$} \\
\hline M0 & & $139(52.3)$ & $127(47.7)$ & 2.737 & 0.255 \\
\hline M1 & & $1(25)$ & $3(75)$ & & \\
\hline MX & & $45(44.6)$ & $56(55.4)$ & & \\
\hline \multicolumn{6}{|l|}{ Pathologic Stage } \\
\hline stage I-II & & $117(45.3)$ & $141(54.7)$ & 7.651 & $0.022^{*}$ \\
\hline stage III-IV & & $56(62.2)$ & $34(37.8)$ & & \\
\hline NA & & $12(52.2)$ & $11(47.8)$ & & \\
\hline
\end{tabular}




\section{A GSE77314}

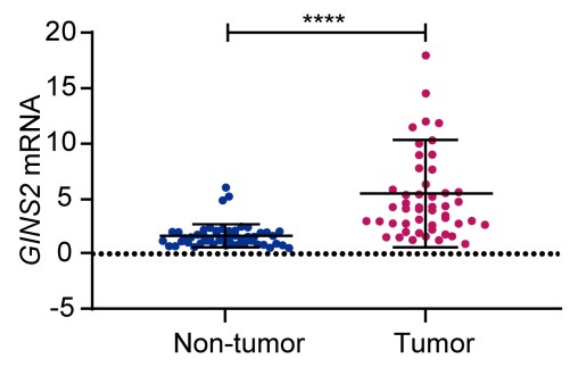

GSE25097

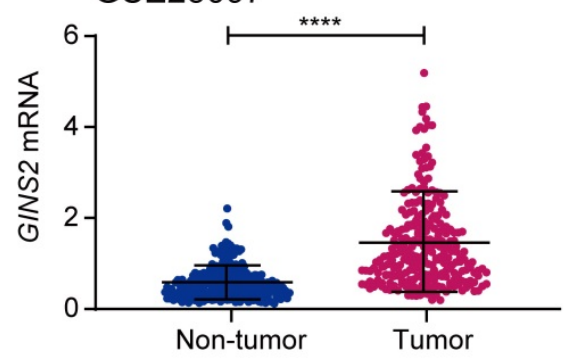

B

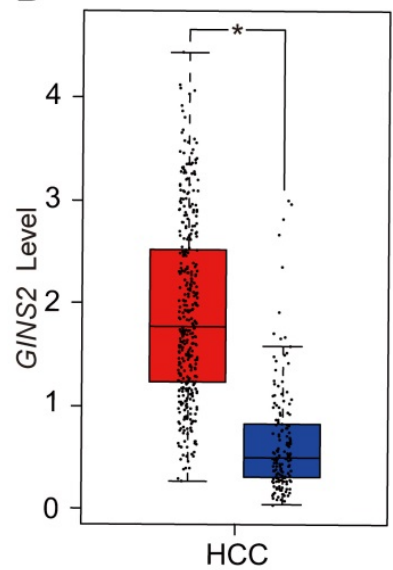

(num $(T)=369$,red; num $(N)=160$,blue)

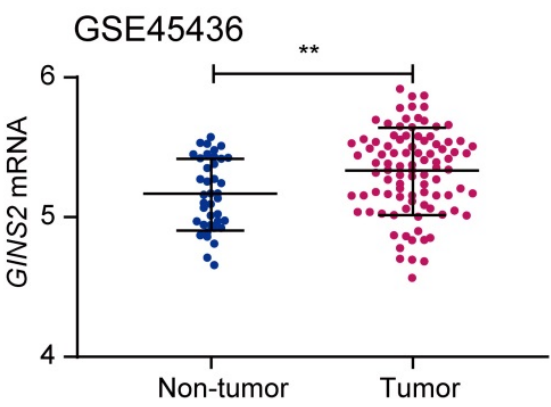

GSE14520

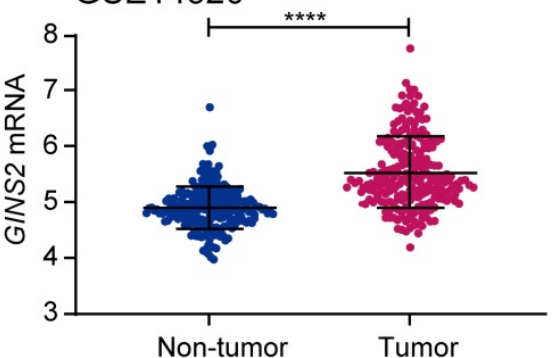

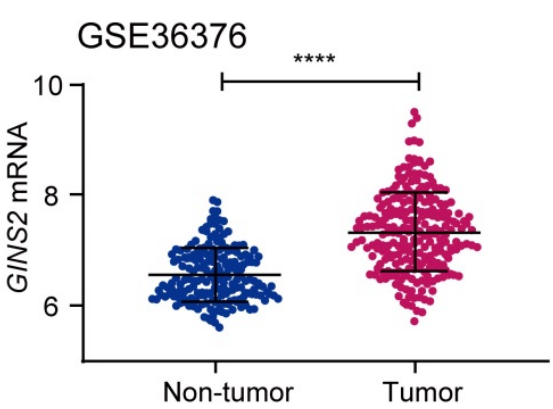

TCGA

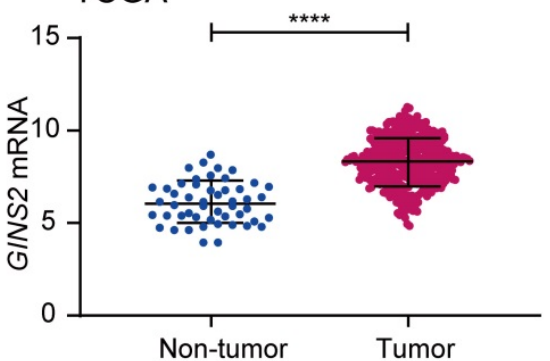

C

Validation cohort

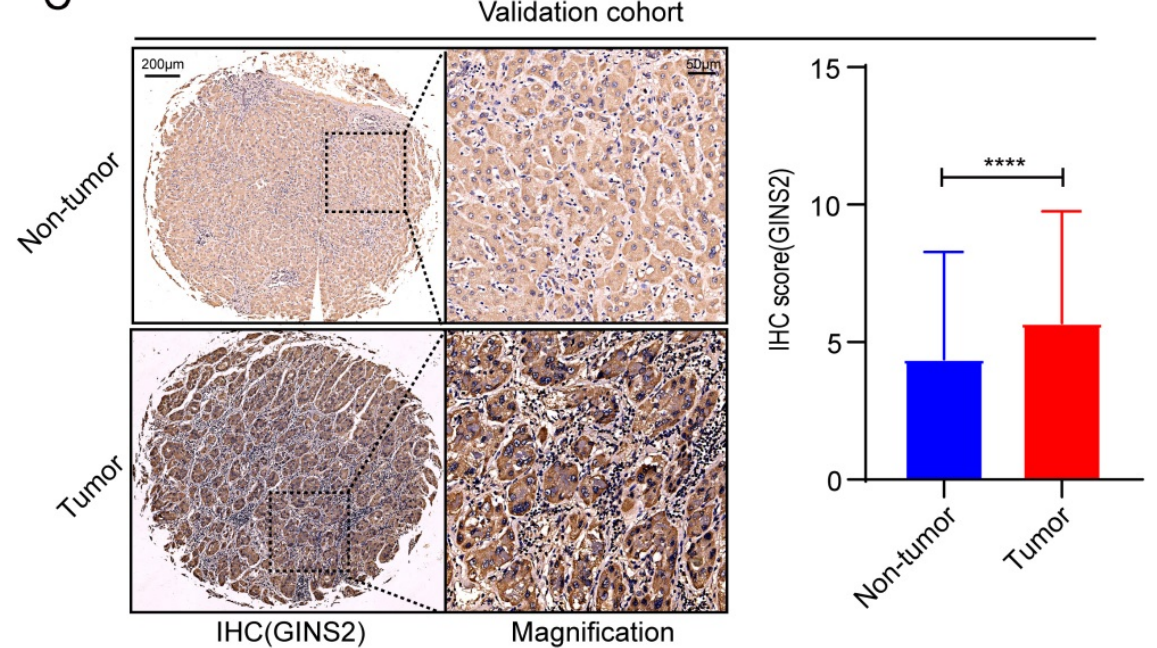

D

OS

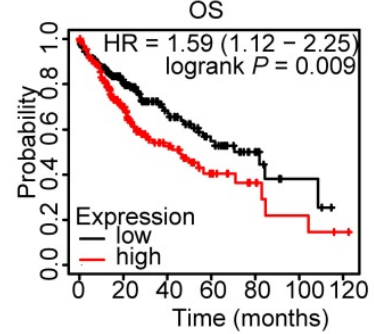

DSS

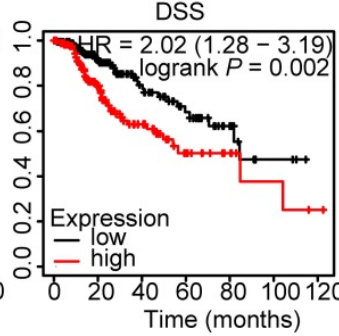

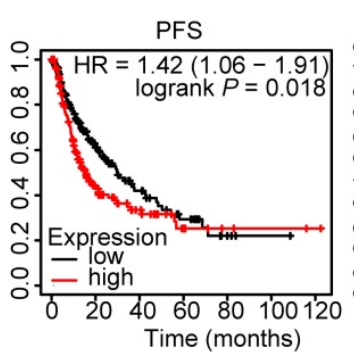

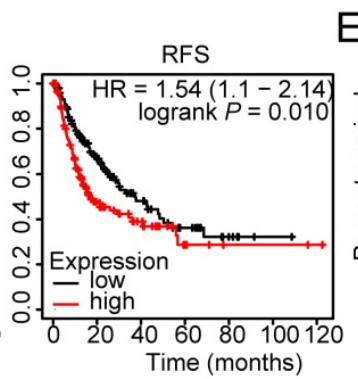

E

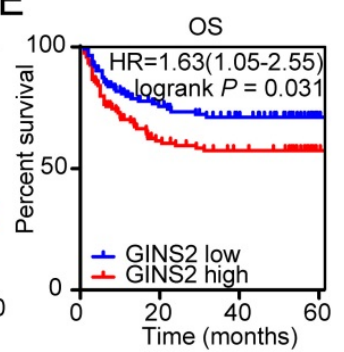

Figure 1. Up-regulated GINS2 in tumorous tissues and predicts poorer prognoses of HCC patients. A. GINS2 mRNA expression levels between tumor and non-tumor tissues of HCC patients of GEO series and TCGA database by Student's t-test or Mann-Whitney U test. B. Box plot of GINS2 levels in tumor and adjacent tissues from the GEPIA database. C. Representative immunohistochemistry staining and statistical results reveal the protein levels of GINS2 in the validation cohort. D. Survival analyses of HCC cohort from TCGA database grouped by GINS2 expression utilizing the K-M plotter tool. OS, overall survival; DSS, disease specific survival; PFS, progression-free survival; RFS, Relapse-free survival. E. Kaplan-Meier survival analysis of the relationship between GINS2 protein expression and OS in the validation cohort. *, $P<0.05, * *, P<0.01, * * *$, $P<0.001, * * * *, P<0.0001$.

\section{GINS2 is vital in the processes of cell cycle and DNA replication}

To unravel the underlying role of GINS2 in liver carcinogenesis and tumor progression, we compared
DEGs in the TCGA dataset between GINS2 high and GINS2 ${ }^{\text {low }}$ groups determined by the GINS2 median cutoff. KEGG pathway and GO analysis were utilized to explore the function of these DEGs (the cutoff criteria were $\log 2(\mathrm{FC})>1$ ). Within these DEGs, 421 
genes were upregulated, and 280 genes were downregulated (Figure 2A). KEGG pathway analysis demonstrated that the upregulated DEGs were mainly enriched in cell cycle and DNA replication signaling pathways (Figure 2B). GO analysis hinted that biological processes involving these upregulated DEGs were cell division, DNA replication and mitotic nuclear division, processes that are related to tumorigenesis and growth (Figure 2C). KEGG pathway analysis also demonstrated that the downregulated DEGs were mainly about metabolic pathways, and the enriched biological processes included oxidation-reduction processes (Figure S3A and $\mathrm{S} 3 \mathrm{~B}$ ). The interactive association of these upregulated DEGs and corresponding term clusters were shown by ClueGO to decipher the biological processes and associations of functionally grouped genes (Figure 2D). The interaction network of DEGs included 139 representative terms and 446 term connections. The results revealed that the significantly enriched biological processes were cell cycle, DNA replication, and processes that have been deemed essential preparations for cell proliferation.

GSEA analysis was also utilized to identify the pathways significantly correlated with GINS2 levels (GINS2 ${ }^{\text {high }}$ vs. GINS2 ${ }^{\text {low}}$ ). The results demonstrated that the gene sets related with high GINS2 expression were enriched in cell cycle, cell division, chromosome separation and DNA replication (Figure 3A, Figure S4A). To further explore the associations between
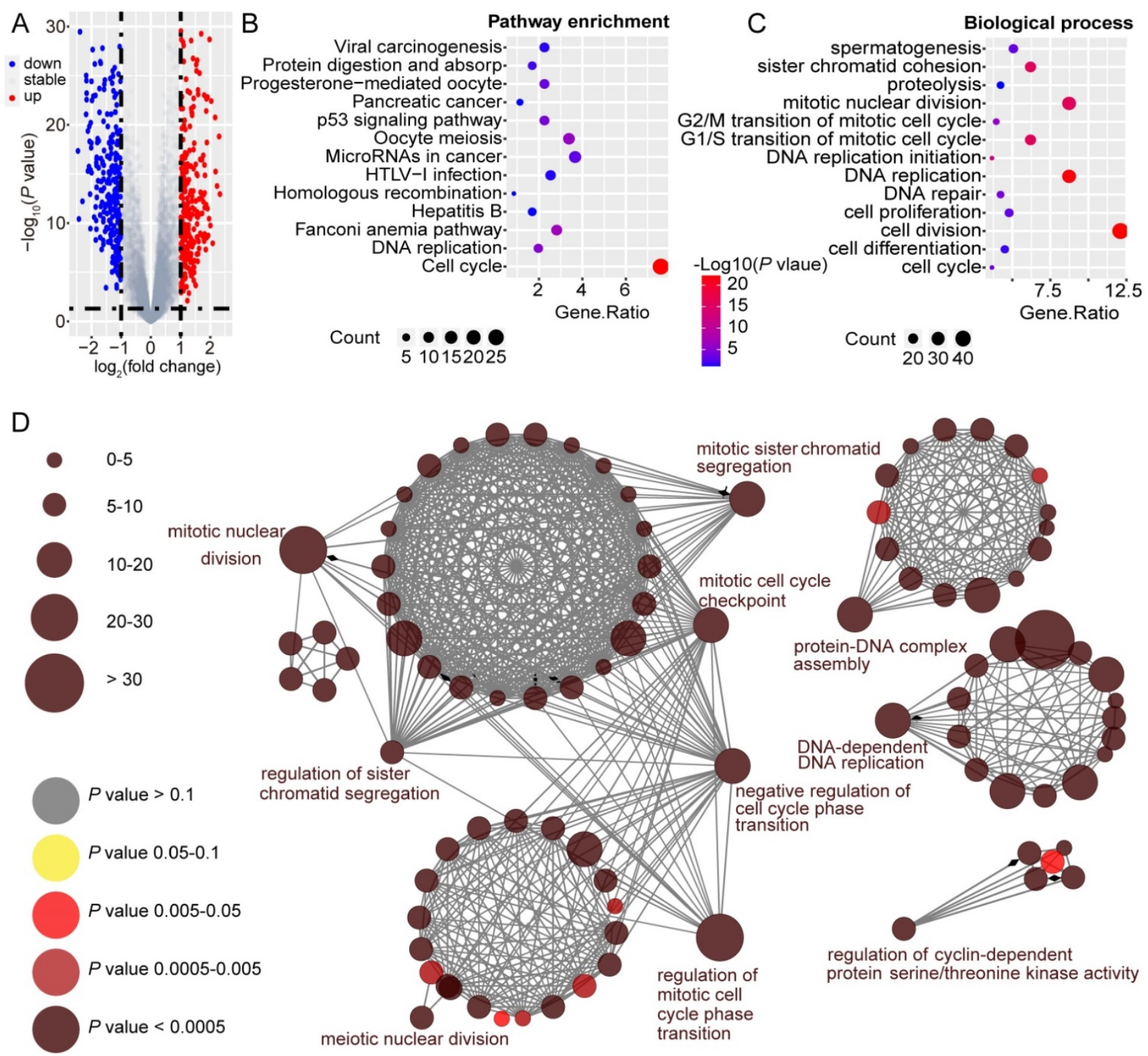

Figure 2. GINS2 is vital in the processes of cell cycle and DNA replication. A. Volcano plot indicates DEGs by comparing GINS2-high and GINS2-low samples from the TCGA database. Blue dot, down-regulated DEGs; red dot, up-regulated DEGs. B/C. Pathway enrichment and biological process of 412 up-regulated DEGs by KEGG analysis. D. Visualization of the interaction network of DEGs by Cytoscape (Cluego module). Node size indicates the mapped gene number; the node color schedule represents the $P$ value. 
GINS2 and cell cycle processes in HCC, a panel of genes related to the cell cycle was compared between the two GINS2 expression groups (GINS2 high vs. GINS2 ${ }^{\text {low }}$ ), and the results indicated that most of these genes were upregulated in the GINS2 ${ }^{\text {high }}$ group. Furthermore, CDK1, which acts as a key factor in the process of the eukaryotic cell cycle by regulating the centrosome cycle and mitotic onset; $C D C 25 \mathrm{~A}$, which takes effect by inducing mitotic progression; and other genes related to the cell cycle (e.g., CDC45, $M C M 3$ and $C D C 25 C$ ) and DNA replication (e.g., CDT1, CHAF1A, and FEN1) were positively correlated with the expression of GINS2 (Figure 3C, Figure S4B), which further implied the significant role of GINS2 in liver carcinogenesis and tumor progression.
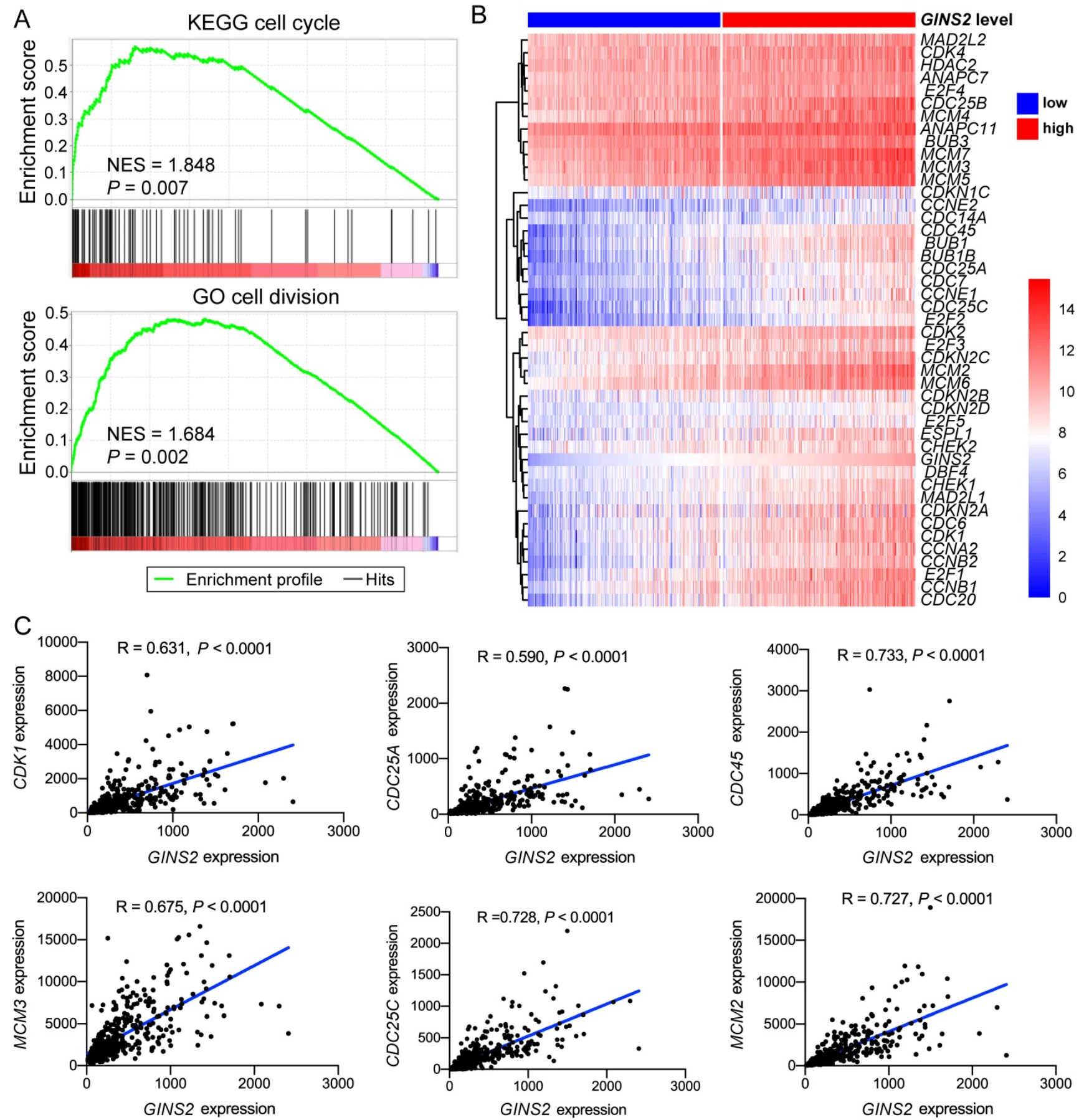

Figure 3. Associations between GINS2 and key genes related to cell cycle in HCC. A. GSEA analysis prompts GINS2 is positively related to cell cycle and cell division. B. Heatmap of genes related to cell cycle grouped by GINS2-high and GINS2-low samples. C. Pearson correlation analysis of GINS2 and key genes involved in cell cycle. 


\section{GINS2 and its co-expressed genes show significant associations with the infiltrating abundance of immune cells in HCC}

Immune cells comprise the most of tumor microenvironment (TME) and somehow participate in tumor cell proliferation and development [23-25]. Therefore, we sought to explore whether the transcription level of GINS2 was associated with the infiltrating abundance of immune cells in HCC. The results revealed that GINS2 levels was positively associated with the abundance of different infiltrating immune cells, including $\mathrm{B}$ cells, $\mathrm{CD}^{+} \mathrm{T}$ cells, macrophages, neutrophils, and dendritic cells (DCs), in HCC (Figure 4A). Moreover, GINS2 co-expressed and cell cycle-related genes, such as CDK1, CDC25A, CDC45, MCM3, CDC25C and MCM2, were also shown positive correlation with the abundance of infiltrating $\mathrm{B}$ cells, $\mathrm{CD}^{+} \mathrm{T}$ cells, $\mathrm{CD}^{+} \mathrm{T}$ cells, macrophages, neutrophils and DCs in HCC (Figure 4B-G). Altogether, these above evidences suggest that GINS2 and its co-expressed genes may have an influence in the immune response in TME by affecting immune infiltrating abundance, especially those of B cells, CD8+ T cells, macrophages and DCs.
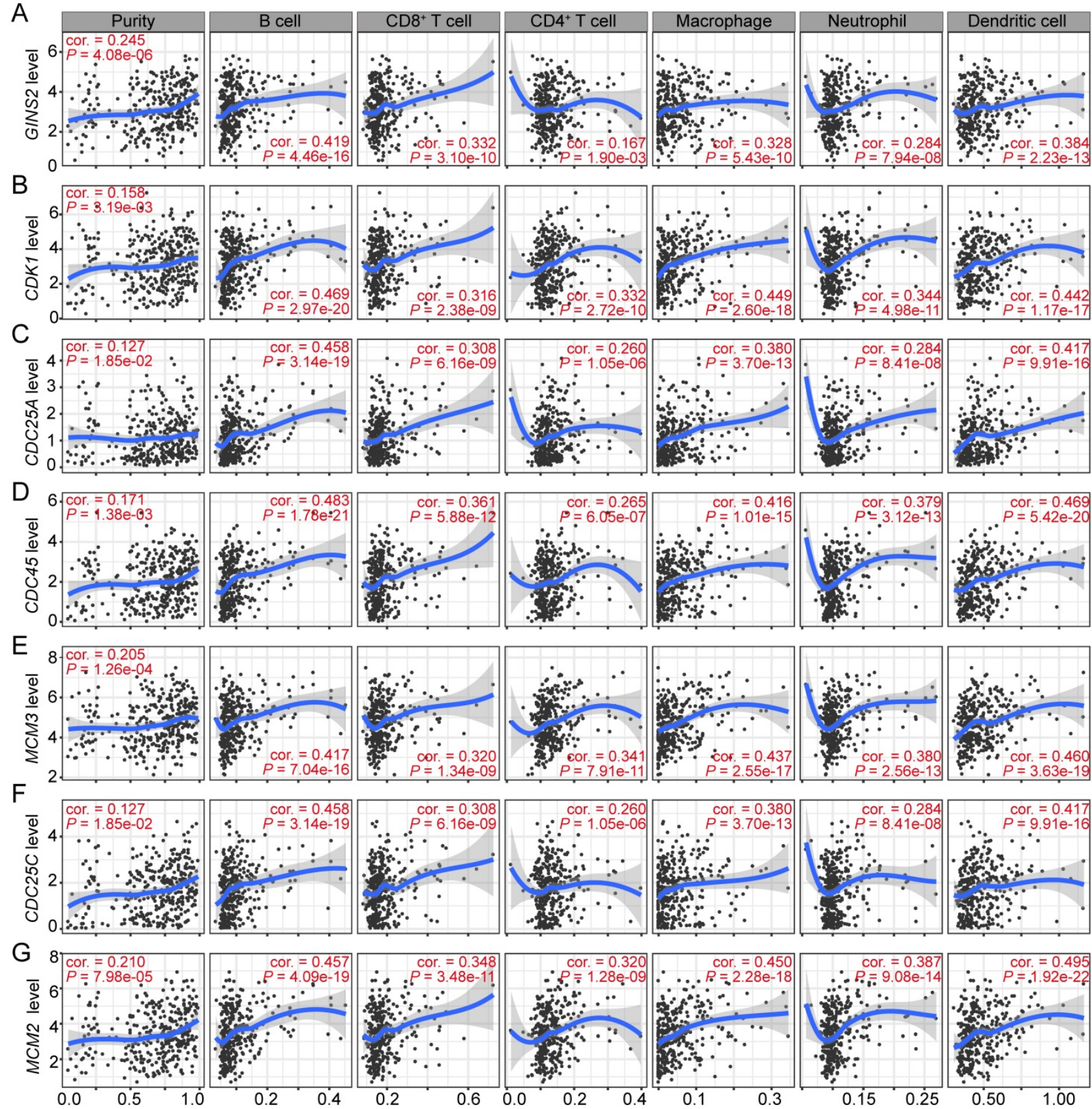

Figure 4. Significant correlations between GINS2 and its co-expressed cell cycle related genes and immunocytes infiltration levels in HCC. Associations between GINS2 (A), CDK1 (B), CDC25A (C), CDC45 (D), MCM3 (E), CDC25C (F), MCM2 (G) expression and diverse immune cells infiltration in HCC. Cor, R value of Spearman's correlation. 
Table 2. Correlation analysis between GINS2 and related gene markers of immune cells in TIMER.

\begin{tabular}{|c|c|c|c|c|c|}
\hline \multirow[t]{3}{*}{ Description } & \multirow[t]{3}{*}{ Gene markers } & \multicolumn{4}{|l|}{ LIHC } \\
\hline & & \multicolumn{2}{|l|}{ None } & \multicolumn{2}{|l|}{ Purity } \\
\hline & & Cor & $\mathrm{P}$ & Cor & $\mathrm{P}$ \\
\hline \multirow[t]{2}{*}{$\mathrm{CD}^{+} \mathrm{T}$ cell } & CD8A & 0.131 & $1.17 \mathrm{E}-02^{*}$ & 0.268 & 4.37E-07* \\
\hline & CD8B & 0.167 & $1.26 \mathrm{E}-03^{*}$ & 0.296 & $2.01 \mathrm{E}-08^{*}$ \\
\hline \multirow[t]{3}{*}{$\mathrm{T}$ cell(general) } & CD3D & 0.221 & $1.82 \mathrm{E}-05^{*}$ & 0.371 & $1.05 \mathrm{E}-12^{*}$ \\
\hline & CD3E & 0.082 & $1.14 \mathrm{E}-01$ & 0.259 & $1.12 \mathrm{E}-06^{*}$ \\
\hline & CD2 & 0.114 & $2.79 \mathrm{E}-02^{*}$ & 0.279 & $1.42 \mathrm{E}-07^{*}$ \\
\hline \multirow[t]{2}{*}{ B cell } & CD19 & 0.162 & $1.79 \mathrm{E}-03^{*}$ & 0.255 & $1.66 \mathrm{E}-06^{*}$ \\
\hline & CD79A & 0.060 & $2.48 \mathrm{E}-01$ & 0.191 & $3.55 \mathrm{E}-04^{*}$ \\
\hline \multirow[t]{2}{*}{ Monocyte } & CD86 & 0.203 & $8.65 \mathrm{E}-05^{*}$ & 0.374 & $7.02 \mathrm{E}-13^{*}$ \\
\hline & CD115(CSF1R) & 0.090 & 8.48E-02 & 0.249 & $2.71 \mathrm{E}-06^{*}$ \\
\hline \multirow[t]{3}{*}{ TAM } & CCL2 & 0.011 & $8.29 \mathrm{E}-01$ & 0.145 & $6.80 \mathrm{E}-03^{*}$ \\
\hline & CD68 & 0.078 & $1.34 \mathrm{E}-01$ & 0.180 & $7.79 \mathrm{E}-04^{*}$ \\
\hline & IL10 & 0.120 & $2.07 \mathrm{E}-02^{*}$ & 0.248 & $3.24 \mathrm{E}-06^{*}$ \\
\hline \multirow[t]{3}{*}{ M1 Macrophage } & INOS(NOS2) & -0.002 & $9.76 \mathrm{E}-01$ & 0.007 & 8.93E-01 \\
\hline & IRF5 & 0.345 & $7.81 \mathrm{E}-12^{*}$ & 0.348 & $2.76 \mathrm{E}-11^{*}$ \\
\hline & COX2(PTGS2) & -0.020 & 6.96E-01 & 0.119 & 2.77E-02* \\
\hline \multirow[t]{3}{*}{ M2 Macrophage } & CD163 & 0.025 & 6.35E-01 & 0.154 & $4.21 \mathrm{E}-03^{*}$ \\
\hline & VSIG4 & 0.065 & 2.09E-01 & 0.199 & $2.03 \mathrm{E}-04^{*}$ \\
\hline & MS4A4A & 0.029 & 5.80E-01 & 0.176 & $1.01 \mathrm{E}-03^{*}$ \\
\hline \multirow[t]{3}{*}{ Neutrophils } & CD66b(CEACAM8) & 0.078 & $1.35 \mathrm{E}-01$ & 0.120 & $2.62 \mathrm{E}-02^{*}$ \\
\hline & CD11b(ITGAM) & 0.272 & $1.13 \mathrm{E}-07^{*}$ & 0.378 & $3.89 \mathrm{E}-13^{*}$ \\
\hline & CCR7 & -0.027 & $6.02 \mathrm{E}-01$ & 0.127 & $1.85 \mathrm{E}-02^{*}$ \\
\hline \multirow[t]{7}{*}{ Natural Killer cell } & KIR2DL1 & -0.028 & $5.95 \mathrm{E}-01$ & -0.044 & 4.13E-01 \\
\hline & KIR2DL3 & 0.116 & $2.53 \mathrm{E}-02^{*}$ & 0.157 & $3.53 \mathrm{E}-03^{*}$ \\
\hline & KIR2DL4 & 0.191 & $2.09 \mathrm{E}-04^{*}$ & 0.230 & $1.58 \mathrm{E}-05^{*}$ \\
\hline & KIR3DL1 & -0.002 & $9.65 \mathrm{E}-01$ & 0.008 & 8.79E-01 \\
\hline & KIR3DL2 & 0.049 & 3.43E-01 & 0.100 & $6.36 \mathrm{E}-02$ \\
\hline & KIR3DL3 & 0.000 & 9.92E-01 & 0.014 & $8.00 \mathrm{E}-01$ \\
\hline & KIR2DS4 & 0.036 & 4.94E-01 & 0.032 & 5.53E-01 \\
\hline \multirow[t]{7}{*}{ Dendritic cell } & HLA-DPB1 & 0.130 & $1.24 \mathrm{E}-02$ & 0.274 & $2.38 \mathrm{E}-07^{*}$ \\
\hline & HLA-DQB1 & 0.145 & $5.19 \mathrm{E}-03^{*}$ & 0.288 & $5.03 \mathrm{E}-08^{*}$ \\
\hline & HLA-DRA & 0.110 & $3.43 \mathrm{E}-02^{*}$ & 0.247 & $3.31 \mathrm{E}-06^{*}$ \\
\hline & HLA-DPA1 & 0.090 & 8.32E-02 & 0.236 & $9.70 \mathrm{E}-06^{*}$ \\
\hline & BDCA-1(CD1C) & -0.026 & 6.13E-01 & 0.083 & $1.22 \mathrm{E}-01$ \\
\hline & BDCA-4(NRP1) & 0.058 & $2.65 \mathrm{E}-01$ & 0.088 & $1.04 \mathrm{E}-01$ \\
\hline & CD11c(ITGAX) & 0.194 & $1.75 \mathrm{E}-04^{*}$ & 0.335 & $1.71 \mathrm{E}-10^{*}$ \\
\hline \multirow[t]{5}{*}{ Th1 cell } & T-bet(TBX21) & 0.013 & 8.03E-01 & 0.134 & $1.25 \mathrm{E}-02^{*}$ \\
\hline & STAT4 & 0.173 & $8.59 \mathrm{E}-04^{*}$ & 0.252 & $2.22 \mathrm{E}-06^{*}$ \\
\hline & STAT1 & 0.249 & $1.31 \mathrm{E}-06^{*}$ & 0.305 & $7.48 \mathrm{E}-09^{*}$ \\
\hline & IFN-y(IFNG) & 0.221 & $1.77 \mathrm{E}-05^{*}$ & 0.310 & $3.88 \mathrm{E}-09^{*}$ \\
\hline & TNF-a(TNF) & 0.149 & $4.12 \mathrm{E}-03^{*}$ & 0.289 & $4.88 \mathrm{E}-08^{*}$ \\
\hline \multirow[t]{4}{*}{ Th2 cell } & GATA3 & 0.102 & $4.92 \mathrm{E}-02^{*}$ & 0.253 & $1.91 \mathrm{E}-06^{*}$ \\
\hline & STAT6 & 0.038 & $4.60 \mathrm{E}-01$ & 0.026 & $6.36 \mathrm{E}-01$ \\
\hline & STAT5A & 0.240 & $2.99 \mathrm{E}-06^{*}$ & 0.306 & $6.77 \mathrm{E}-09^{*}$ \\
\hline & IL13 & 0.123 & $1.75 \mathrm{E}-02^{*}$ & 0.123 & $2.27 \mathrm{E}-02^{*}$ \\
\hline Tfh cell & BCL6 & 0.011 & 8.31E-01 & 0.015 & 7.79E-01 \\
\hline & IL21 & 0.078 & $1.34 \mathrm{E}-01$ & 0.130 & $1.58 \mathrm{E}-02^{*}$ \\
\hline Th17 cell & STAT3 & 0.030 & 5.61E-01 & 0.076 & $1.61 \mathrm{E}-01$ \\
\hline & IL17A & 0.001 & $9.84 \mathrm{E}-01$ & 0.019 & $7.28 \mathrm{E}-01$ \\
\hline Treg cell & FOXP3 & 0.116 & $2.52 \mathrm{E}-02^{*}$ & 0.202 & $1.53 \mathrm{E}-04^{*}$ \\
\hline & CCR8 & 0.203 & $8.53 \mathrm{E}-05^{*}$ & 0.297 & $1.82 \mathrm{E}-08^{*}$ \\
\hline & STAT5B & 0.135 & $9.18 \mathrm{E}-03^{*}$ & 0.103 & $5.68 \mathrm{E}-02$ \\
\hline & TGF $\beta$ (TGFB1) & 0.138 & 7.79E-03* & 0.241 & $6.03 \mathrm{E}-06^{*}$ \\
\hline T cell exhaustion & PD-1(PDCD1) & 0.217 & $2.38 \mathrm{E}-05^{*}$ & 0.340 & $8.68 \mathrm{E}-11^{*}$ \\
\hline & CTLA4 & 0.230 & 7.77E-06* & 0.362 & $3.90 \mathrm{E}-12^{*}$ \\
\hline & LAG3 & 0.292 & $1.16 \mathrm{E}-08^{*}$ & 0.356 & $9.35 \mathrm{E}-12^{*}$ \\
\hline & TIM-3(HAVCR2) & 0.229 & $8.81 \mathrm{E}-06^{*}$ & 0.404 & $5.29 \mathrm{E}-15^{*}$ \\
\hline
\end{tabular}

TIMER: Correlation module analyzes the expression between a pair of specific genes in HCC, together with the Spearman's rho value and estimated statistical significance. Options for partial correlation conditioned on tumor purity are also provided. Cor, R value of Spearman's correlation; None, correlation without adjustment. Purity, correlation adjusted by purity. ${ }^{*} P<0.05$

\section{GINS2 levels and its correlation with immune marker gene sets}

To further explore the potential association between GINS2 gene and different infiltrating immunocytes, we then utilized the TIMER and GEPIA databases to achieve this end. We investigated the associations between transcriptional levels of GINS2 and gene markers of various immune cells or status and the results were demonstrated in Table 2. After equilibrating the effect of tumor purity, transcriptional levels of GINS2 were positively related with most marker gene sets of $\mathrm{CD}^{+} \mathrm{T}$ cells, $\mathrm{T}$ cells (general) and Th1 cells in HCC (Table 2). We also 
analyzed the samples of validation cohort by multiplex immunohistochemistry (mIHC, Figure 5A). The protein levels of GINS2 were positively associated with the number of infiltrating $\mathrm{CD}^{+}{ }^{+} \mathrm{CD} 8^{+} \mathrm{T}$ cells and $\mathrm{CD}^{+}{ }^{+} \mathrm{CD} 4^{+} \mathrm{T}$ cells (Figure 5B-C). To further elucidate the correlation between GINS2 gene and T cell status, we examined the associations between GINS2 levels and markers of activated $\mathrm{T}$ cells, including activated $\mathrm{CD}^{+} \mathrm{T}$ cells and $\mathrm{CD}^{+} \mathrm{T}$ cells (Table 3). After equilibrating the effect of tumor purity, the results revealed that the GINS2 level showed positive correlation with most markers of activated $\mathrm{T}$ cells in HCC.

Among $\mathrm{T}$ cells, $\mathrm{CD}^{+} \mathrm{T}$ cells are the most significant effective anti-tumor cells. After $\mathrm{CD} 8^{+} \mathrm{T}$ cells infiltrate the tumor tissue, they gradually transition into a state of dysfunctional exhaustion under the chronic and continuous stimulation of tumor-related antigens, named $\mathrm{T}$ cell exhaustion, which is a significant mechanism in the weakening of antitumor effects [26]. Interestingly, we noticed significant associations between GINS2 levels and marker sets of Treg cells and T cell exhaustion, such as FOXP3, CCR8, TGF $\beta$, PD-1, CTLA4, LAG3, and TIM-3 (Table 2). FOXP3 acts a pivotal role in Treg cells, in which it impedes the attack of cytotoxic $\mathrm{T}$ cells on cancerous cells [27]. TIM-3, a vital gene that mediates $\mathrm{T}$ cell exhaustion, correlates positively with GINS2 levels, implying that GINS2 gene acts as a crucial role in TIM-3 mediating $\mathrm{T}$ cell exhaustion. Other immunosuppressive molecules, e.g., PD-1 and CTLA4, also showed important associations with GINS2 levels. The above evidences revealed that there is a link between GINS2 gene and T cell exhaustion and that GINS2 may play a pivotal role in immune escape in the TME of HCC.

In addition, the transcriptional levels of most gene markers of TAMs, M1 macrophages, M2 macrophages and natural killer cells had weak or no correlations with GINS2 expression (Table 2). We further verified the associations between GINS2 gene and marker sets of monocytes and TAMs via the

A
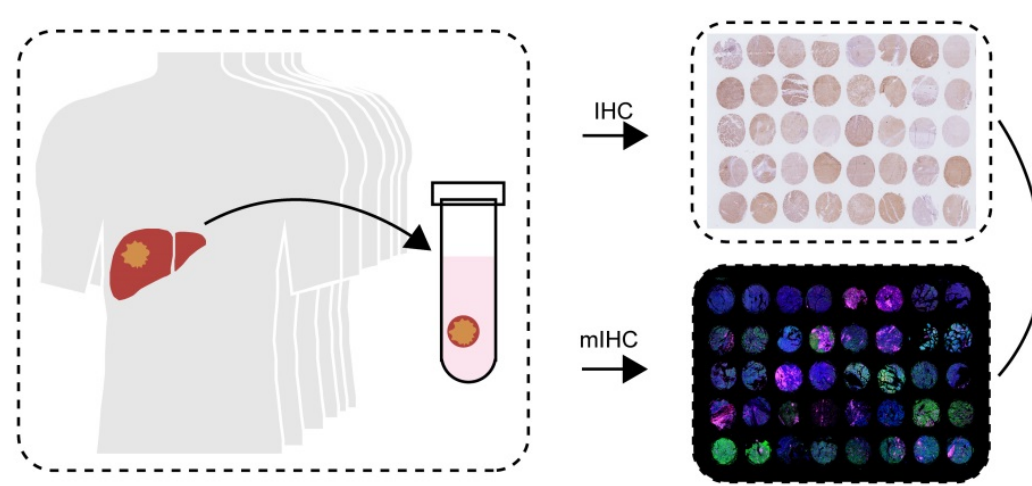

B GINS2 High

GINS2 Low
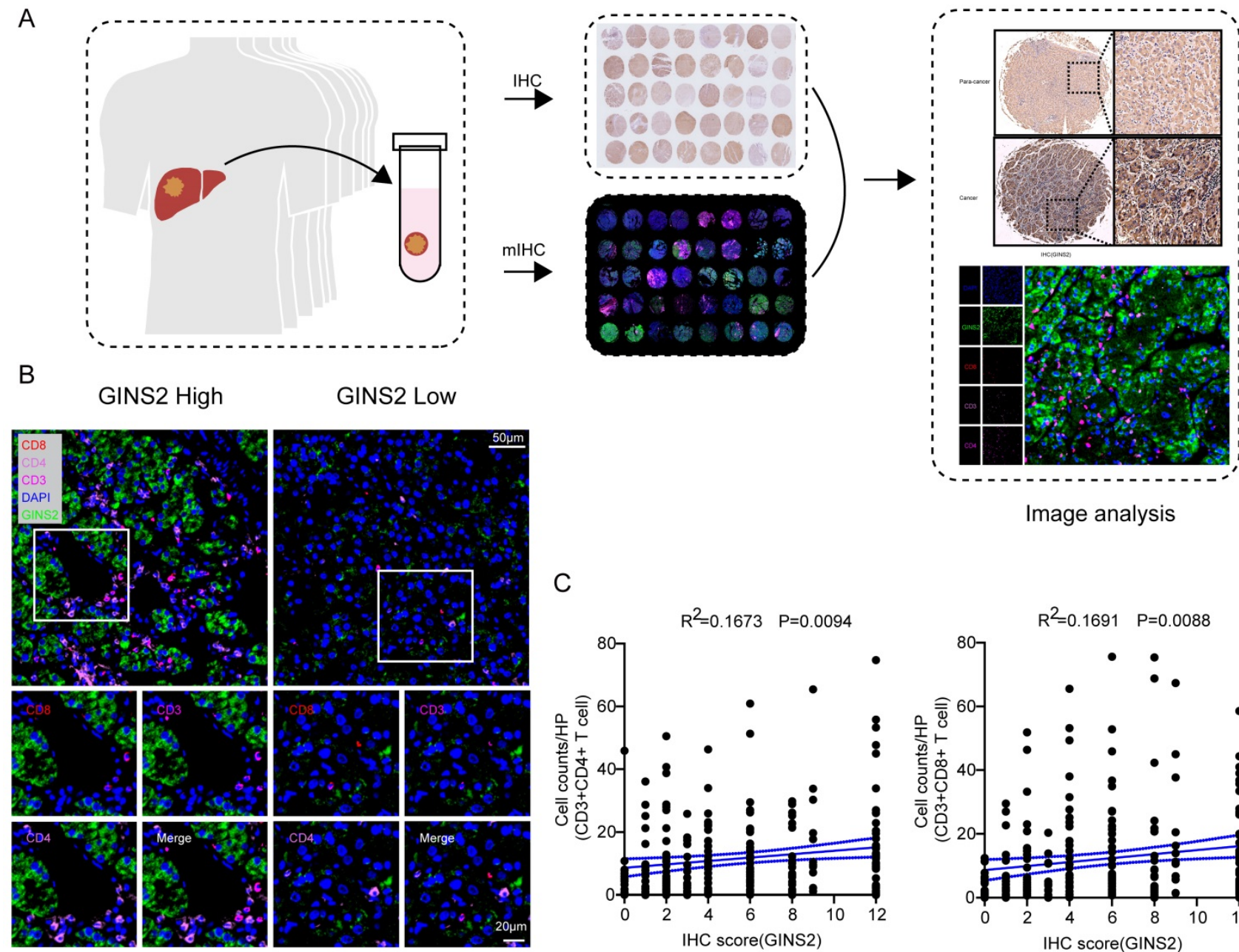

Image analysis

Figure 5. Significant correlations between GINS2 levels and CD3+CD8+ $T$ cells/ CD3 $3^{+} C D 4^{+} T$ cells in the validation cohort. A. Schematic workflow of samples from validation cohort for traditional IHC and mIHC. B. Representative multiplex fluorescent immunohistochemistry images show the features of $C D 3^{+} \mathrm{CD} 8^{+} \mathrm{T}$ cells/CD $3^{+} \mathrm{CD} 4^{+}$ T cells within the tissues of high and low GINS2 expression. C. Correlation analyses of GINS2 protein levels (IHC Score) and infiltration of $C D 3^{+} C D 8^{+} T$ cells and $C D 3^{+} C D 4^{+} T$ cells in the validation cohort. 
GEPIA database. The correlations between GINS2 and markers of monocytes and TAMs were similar with those identified via TIMER database (Table 4). The findings suggest that GINS2 does not exert much influence on the regulation of macrophage polarization in HCC.

\section{Discussion}

DNA replication, an important step in the cell cycle enabling one single tumor cell to split into two, is emerging as an important factor in tumorigenesis and growth [28]. GINS complex subunit 2 (GINS2), a crucial element of the DNA replication complex GINS, takes part in multiple processes of DNA replication [11]. To gain new insights into the underlying roles of GINS2 in liver cancer and its underlying mechanism, we applied a bioinformatic analysis based on public database. Analysis of the transcriptome in samples from 5 GEO datasets and 421 HCC samples from the TCGA database verified that transcriptional levels of GINS2 are significantly elevated in cancerous tissues than in normal tissues, which is line with the results of the IHC staining of 236-patient validation cohort (Figure 1). Furthermore, GINS2 gene reflected the prognosis of HCC patients. The overall survival, disease-free survival, progression-free survival, and relapse-free survival of patients with high-GINS2 expression were worse than those with low-GINS2 expression in the TCGA cohort.

Table 3. Correlation analysis between GINS2 and markers of activated T cells in HCC patients in TIMER.

\begin{tabular}{|c|c|c|c|c|c|c|c|c|c|}
\hline \multirow[t]{2}{*}{ Activated CD8Tcell } & \multicolumn{2}{|l|}{ None } & \multicolumn{2}{|l|}{ Purity } & \multirow{2}{*}{$\begin{array}{l}\text { Activated } \\
\text { CD4Tcell }\end{array}$} & \multicolumn{2}{|l|}{ None } & \multicolumn{2}{|c|}{ Purity } \\
\hline & Cor & $\mathrm{P}$ & Cor & $\mathrm{P}$ & & Cor & $\mathrm{P}$ & Cor & $\mathrm{P}$ \\
\hline ADRM1 & 0.386 & $1.36 \mathrm{e}-14^{*}$ & 0.384 & $1.53 \mathrm{E}-13^{*}$ & AIM2 & 0.187 & $2.87 \mathrm{e}-04^{*}$ & 0.331 & 2.89E-10* \\
\hline AHSA1 & 0.28 & $4.77 \mathrm{e}-08^{*}$ & 0.309 & $4.41 \mathrm{E}-09^{*}$ & BIRC3 & 0.162 & $1.73 e-03^{*}$ & 0.230 & $1.58 \mathrm{E}-05^{*}$ \\
\hline C1GALT1C1 & 0.132 & $1.11 \mathrm{e}-02^{*}$ & 0.161 & $2.69 \mathrm{E}-03^{*}$ & BRIP1 & 0.525 & $1.00 \mathrm{e}-27^{*}$ & 0.511 & 2.63E- $24^{*}$ \\
\hline ССТ6В & -0.121 & $1.93 \mathrm{e}-02 *$ & -0.139 & $9.80 \mathrm{E}-03$ & CCL20 & 0.121 & $2.02 \mathrm{e}-02^{*}$ & 0.146 & $6.58 \mathrm{E}-03^{*}$ \\
\hline CD37 & 0.122 & $1.88 \mathrm{e}-02^{*}$ & 0.310 & $4.21 \mathrm{E}-09^{*}$ & CCL4 & 0.112 & $3.17 \mathrm{e}-02^{*}$ & 0.264 & $6.71 \mathrm{E}-07^{*}$ \\
\hline CD3D & 0.221 & $1.82 \mathrm{e}-05^{*}$ & 0.371 & $1.05 \mathrm{E}-12^{*}$ & CCL5 & 0.129 & $1.27 \mathrm{e}-02^{*}$ & 0.278 & 1.54E-07* \\
\hline CD3E & 0.082 & $1.14 \mathrm{e}-01$ & 0.259 & $1.12 \mathrm{E}-06^{*}$ & CCNB1 & 0.75 & $2.72 \mathrm{e}-68^{*}$ & 0.742 & $1.91 \mathrm{E}-61^{*}$ \\
\hline CD3G & 0.102 & $4.97 \mathrm{e}-02^{*}$ & 0.214 & $6.39 \mathrm{E}-05^{*}$ & CCR7 & -0.027 & $6.02 \mathrm{e}-01$ & 0.127 & $1.85 \mathrm{E}-02^{*}$ \\
\hline CD69 & 0.02 & $6.97 \mathrm{e}-01$ & 0.159 & 3.07E-03* & DUSP2 & 0.178 & $5.88 \mathrm{e}-04^{*}$ & 0.299 & 1.42E-08* \\
\hline CD8A & 0.131 & $1.17 \mathrm{e}-02^{*}$ & 0.268 & 4.37E-07* & ESCO2 & 0.689 & $0.00 \mathrm{e}+00^{*}$ & 0.678 & 1.04E-47* \\
\hline CETN3 & 0.35 & $3.83 e-12^{*}$ & 0.326 & $5.68 \mathrm{E}-10^{*}$ & ETS1 & -0.034 & $5.15 \mathrm{e}-01$ & 0.071 & $1.88 \mathrm{E}-01$ \\
\hline CSE1L & 0.526 & $0.00 \mathrm{e}+00^{*}$ & 0.515 & $9.85 \mathrm{E}-25^{*}$ & EXO1 & 0.701 & $0.00 \mathrm{E}+00^{*}$ & 0.742 & 1.32E-75* \\
\hline GEMIN6 & 0.362 & $6.27 \mathrm{e}-13^{*}$ & 0.334 & $1.92 \mathrm{E}-10^{*}$ & EXOC6 & 0.327 & $1.46 \mathrm{e}-10^{*}$ & 0.313 & 2.80E-09* \\
\hline GNLY & 0.093 & 7.37e-02 & 0.168 & $1.71 \mathrm{E}-03^{*}$ & IARS & 0.352 & $3.78 \mathrm{e}-12^{*}$ & 0.352 & 1.69E- $11^{*}$ \\
\hline GPT2 & -0.229 & $8.73 e-06^{*}$ & -0.231 & $1.41 \mathrm{E}-05^{*}$ & ITK & 0.033 & $5.29 \mathrm{e}-01$ & 0.186 & $5.22 \mathrm{E}-04^{*}$ \\
\hline GZMA & 0.009 & $7.59 \mathrm{e}-02$ & 0.223 & $2.90 \mathrm{E}-05^{*}$ & KIF11 & 0.762 & $1.57 \mathrm{e}-71^{*}$ & 0.749 & 3.52E-63* \\
\hline GZMH & 0.051 & $3.26 \mathrm{e}-01$ & 0.134 & $1.30 \mathrm{E}-02^{*}$ & KNTC1 & 0.754 & $0.00 \mathrm{e}+00^{*}$ & 0.736 & 4.62E-60* \\
\hline GZMK & 0.014 & 7.91e-01 & 0.165 & $2.15 \mathrm{E}-03^{*}$ & NUF2 & 0.753 & $0.00 \mathrm{e}+00^{*}$ & 0.741 & 3.38E-61* \\
\hline IL2RB & 0.15 & $3.72 \mathrm{e}-03^{*}$ & 0.290 & $4.16 \mathrm{E}-08^{*}$ & PRC1 & 0.755 & $0.00 \mathrm{e}+00^{*}$ & 0.739 & 6.77E-61* \\
\hline LCK & 0.103 & $4.73 \mathrm{e}-02^{*}$ & 0.268 & 4.30E-07* & PSAT1 & 0.143 & $5.79 \mathrm{e}-03^{*}$ & 0.148 & $6.05 \mathrm{E}-03^{*}$ \\
\hline MPZL1 & 0.386 & $1.21 \mathrm{e}-14^{*}$ & 0.414 & $1.04 \mathrm{E}-15^{*}$ & RGS1 & 0.223 & $1.50 \mathrm{e}-05^{*}$ & 0.363 & 3.62E-12* \\
\hline NKG7 & 0.007 & $1.64 \mathrm{e}-01$ & 0.173 & $1.26 \mathrm{E}-03^{*}$ & RTKN2 & 0.603 & $3.67 \mathrm{e}-38^{*}$ & 0.588 & $2.05 \mathrm{E}-33^{*}$ \\
\hline PIK3IP1 & -0.01 & $8.42 \mathrm{e}-01$ & 0.031 & 5.65E-01 & SAMSN1 & 0.177 & $6.48 \mathrm{e}-04^{*}$ & 0.356 & 9.15E-12* \\
\hline PTRH2 & 0.228 & $1.01 \mathrm{e}-05^{*}$ & 0.230 & $1.58 \mathrm{E}-05^{*}$ & SELL & 0.072 & $1.67 \mathrm{e}-01$ & 0.189 & $4.05 \mathrm{E}-04^{*}$ \\
\hline TIMM13 & 0.252 & $8.91 \mathrm{e}-07^{*}$ & 0.243 & $4.87 \mathrm{E}-06^{*}$ & TRAT1 & 0.011 & $8.32 \mathrm{e}-01$ & 0.153 & $4.29 \mathrm{E}-03^{*}$ \\
\hline ZAP70 & 0.07 & $1.79 \mathrm{e}-01$ & 0.207 & $1.05 \mathrm{E}-04^{*}$ & & & & & \\
\hline
\end{tabular}

Table 4. Correlation analysis between GINS2 and related gene markers of monocyte and macrophages in GEPIA.

\begin{tabular}{|c|c|c|c|c|c|}
\hline \multirow[t]{3}{*}{ Description } & \multirow[t]{3}{*}{ Gene markers } & \multicolumn{4}{|l|}{$\mathrm{HCC}$} \\
\hline & & \multicolumn{2}{|l|}{ Tumor } & \multicolumn{2}{|c|}{ Normal } \\
\hline & & $\mathrm{R}$ & $P$ & $\mathrm{R}$ & $P$ \\
\hline \multirow[t]{2}{*}{ Monocyte } & CD86 & 0.18 & $6.20 \mathrm{E}-04^{*}$ & 0.056 & $7.00 \mathrm{E}-01$ \\
\hline & CD115(CSF1R) & 0.12 & $2.30 \mathrm{E}-02^{*}$ & 0.059 & $6.80 \mathrm{E}-01$ \\
\hline \multirow[t]{3}{*}{ TAM } & CCL2 & 0.028 & $5.90 \mathrm{E}-01$ & 0.076 & $6.00 \mathrm{E}-01$ \\
\hline & CD68 & 0.05 & 3.40E-01 & 0.012 & $9.40 \mathrm{E}-01$ \\
\hline & IL10 & 0.16 & $2.50 \mathrm{E}-03^{*}$ & 0.23 & 1.10E-01 \\
\hline \multirow[t]{3}{*}{ M1 Macrophage } & INOS (NOS2) & -0.0082 & $8.80 \mathrm{E}-01$ & 0.58 & $1.20 \mathrm{E}-05^{*}$ \\
\hline & IRF5 & 0.26 & 3.50E-07* & 0.095 & 5.10E-01 \\
\hline & COX2(PTGS2) & -0.0081 & $8.80 \mathrm{E}-01$ & -0.024 & $8.70 \mathrm{E}-01$ \\
\hline \multirow[t]{3}{*}{ M2 Macrophage } & CD163 & 0.12 & $2.60 \mathrm{E}-02^{*}$ & -0.09 & $5.30 \mathrm{E}-01$ \\
\hline & VSIG4 & 0.11 & $3.20 \mathrm{E}-02^{*}$ & -0.085 & $5.60 \mathrm{E}-01$ \\
\hline & MS4A4A & 0.083 & 1.10E-01 & 0.021 & $8.80 \mathrm{E}-01$ \\
\hline
\end{tabular}

R. Value of Pearson's correlation; $P$. Statistical significance. ${ }^{*} P<0.05$. 
High expression of GINS2 can cause an unfavorable prognosis in male HCC patients. However, GINS2 has different effects on the outcome of HCC cohorts of different ethnicities. High levels of GINS2 can cause a poor prognosis in Asian HCC patients but cannot affect Caucasians. Additionally, our studies demonstrated that high GINS2 levels displayed poor OS and DSS in patients without a history of alcohol consumption or hepatitis virus infection. Thus, our results hinted that GINS2 might serve as a prognostic biomarker in HCC. In an attempt to determine underlying biological processes potentially responsible for the poor prognosis tied with GINS2 expression, we found that GINS2 gene was related with a series process of cell proliferation, say, cell cycle, DNA replication and cell division processes, in HCC. These processes affect tumor growth and the prognosis of hepatocellular carcinoma. Indeed, GINS2, as a novel unraveled oncogene, has been reported to affect cell viability, apoptosis, and cell cycle progression in pancreatic cancer [29]. Knockdown of GINS2 impairs cell proliferation and enhances apoptosis in non-small-cell lung cancer (NSCLC) [30]. Given these direct and indirect effects of GINS2, we cautiously conclude that GINS2 overexpression contributes to unfavorable prognosis in HCC patients and the underlying function of GINS2 in HCC was correlated with DNA replication and cell cycle.

Our study also found that GINS2 levels was correlated with the levels of diverse infiltrating immunocytes in HCC, which, until now, had not been extensively studied. Our analyses revealed that in $\mathrm{HCC}$, the abundance and gene markers of diverse immunocytes were associated with the GINS2 levels. Specifically, GINS2 expression was related with the levels of infiltrating $\mathrm{B}$ cells, $\mathrm{CD}^{+} \mathrm{T}$ cells, macrophages, neutrophils, and dendritic cells (DCs) in HCC. Meanwhile, GINS2 levels was significantly associated with marker sets of $\mathrm{CD}^{+} \mathrm{T}$ cells, $\mathrm{T}$ cells (general), Th1 cells, Treg cells and T cell exhaustion in HCC. Importantly, our results demonstrated that the levels of GINS2 expression was positively correlated with most gene markers of activated T cells, especially $\mathrm{CD}^{+} \mathrm{T}$ cells, in HCC. CD8 ${ }^{+} \mathrm{T}$ cells, as the prime anti-tumor cells, can destroy cancerous cells by secreting perforin and granzyme $B$ through the Fas/FasL pathway once they contact tumor cells or release IFN- $\gamma$ and TNFa to eliminate tumor cells [2]. However, $\mathrm{CD}^{+} \mathrm{T}$ lymphocytes in TME are often exhausted, which is tightly related to the activation of immune checkpoints (such as PD1 and CTLA4), as has been reported in our studies. Most malignant tumors, including HCC, enhance the expression of inhibitory ligands to get out of the immune response by destroying $\mathrm{T}$ cell function, thus lead to tumor progression. This is the crucial mechanism promoting tumor progression and immune escape. Moreover, the markers of Treg cells, FOXP3, CCR8 and TGF- $\beta$, are positively correlated with GINS2 expression. Treg cells are one of the important subgroups of $\mathrm{CD} 4^{+} \mathrm{T}$ cells. Previous research has reported that as the tumor progresses, the number of Treg cells increases [31]. Treg cells are important immunosuppressive cells, suggesting that HCC patients with high GINS2 expression might have certain degrees of immunosuppression. TAMs can assist cancerous cells in various ways, e.g., promoting tumor cell proliferation, angiogenesis, immune escape, and metastasis [32-35]. However, our results demonstrate that there are no or only weak associations between the levels of GINS2 and marker sets of monocytes, TAMs, M1 macrophages, M2 macrophages and natural killer cells in HCC.

In conclusion, our study suggests that GINS2 is a potential prognostic biomarker for HCC patients and is associated with the abundance of infiltrating immune cells in tumor tissues. Relatively high levels of GINS2 in HCC may indicate a greater risk of poor prognosis, and considering the differences in infiltration levels of immune cells between the groups with high- and low- expression of GINS2, HCC patients with high GINS2 expression may benefit from more accurate immunotherapy strategies.

\section{Supplementary Material}

Supplementary figures and tables.

https://www.jcancer.org/v13p0610s1.pdf

\section{Acknowledgements}

\section{Funding}

This work was supported by grants from The National Natural Science Foundation of China (grant no.81702337 and no.81670514).

\section{Author Contributions}

(I) Conception and design: Yupeng Wang, (II) Administrative support: Xiaoliang Wang, (III) Collection and assembly of data: Dezhen Guo, Chen Qiu, Zhijie Zhou and Chao Xiao, (IV) Data analysis and interpretation: Zuyin Li and Guohe Song, (V) Manuscript writing: All authors, (VI)Final approval of manuscript: All authors.

\section{Competing Interests}

The authors have declared that no competing interest exists. 


\section{References}

1. Bray F, Ferlay J, Soerjomataram I, Siegel RL, Torre LA, Jemal A. Global cancer statistics 2018: GLOBOCAN estimates of incidence and mortality worldwide for 36 cancers in 185 countries. CA Cancer J Clin. 2018; 68: 394-424.

2. Ringelhan M, Pfister D, O'Connor T, Pikarsky E, Heikenwalder M. The immunology of hepatocellular carcinoma. Nat Immunol. 2018; 19: 222-32.

3. Nishikawa H, Koyama S. Mechanisms of regulatory $\mathrm{T}$ cell infiltration in tumors: implications for innovative immune precision therapies. J Immunother Cancer. 2021; 9: e002591.

4. Ficht $\mathrm{X}$, Iannacone M. Immune surveillance of the liver by $\mathrm{T}$ cells. Sci Immunol. 2020; 5: eaba2351.

5. Qiu J, Tang W, Du C. Immune checkpoint inhibitors in patients with recurrent hepatocellular carcinoma after liver transplantation: one case report and literature review. Curr Cancer Drug Targets. 2020;9:720-27.

6. Wang BJ, Bao JJ, Wang JZ, Wang Y, Jiang M, Xing MY, et al. Immunostaining of PD-1/PD-Ls in liver tissues of patients with hepatitis and hepatocellular carcinoma. World J Gastroenterol. 2011; 17: 3322-9.

7. Shi F, Shi M, Zeng Z, Qi RZ, Liu ZW, Zhang JY, et al. PD-1 and PD-L1 upregulation promotes $\mathrm{CD} 8(+) \mathrm{T}$-cell apoptosis and postoperative recurrence in hepatocellular carcinoma patients. Int J Cancer. 2011; 128: 887-96.

8. Kim HD, Song GW, Park S, Jung MK, Kim MH, Kang HJ, et al. Association Between Expression Level of PD1 by Tumor-Infiltrating CD8(+) T Cells and Features of Hepatocellular Carcinoma. Gastroenterology. 2018; 155: 1936-50 e17.

9. Gao Q, Wang XY, Qiu SJ, Yamato I, Sho M, Nakajima Y, et al. Overexpression of PD-L1 significantly associates with tumor aggressiveness and postoperative recurrence in human hepatocellular carcinoma. Clin Cancer Res. 2009; 15: 971-9.

10. Takayama Y, Kamimura Y, Okawa M, Muramatsu S, Sugino A, Araki H. GINS, a novel multiprotein complex required for chromosomal DNA replication in budding yeast. Genes Dev. 2003; 17: 1153-65.

11. Kamada K, Kubota Y, Arata T, Shindo Y, Hanaoka F. Structure of the human GINS complex and its assembly and functional interface in replication initiation. Nat Struct Mol Biol. 2007; 14: 388-96.

12. Thomassen $M$, Tan $Q$, Kruse TA. Gene expression meta-analysis identifies chromosomal regions and candidate genes involved in breast cancer metastasis. Breast Cancer Res Treat. 2009; 113: 239-49.

13. Zhang M, He S, Ma X, Ye Y, Wang G, Zhuang J, et al. GINS2 affects cell viability, cell apoptosis, and cell cycle progression of pancreatic cancer cells via MAPK/ERK pathway. J Cancer. 2020; 11: 4662-70.

14. Chi F, Wang Z, Li Y, Chang N. Knockdown of GINS2 inhibits proliferation and promotes apoptosis through the p53/GADD45A pathway in non-small-cell lung cancer. Biosci Rep. 2020; 40:BSR20193949.

15. Rantala JK, Edgren H, Lehtinen L, Wolf M, Kleivi K, Vollan HK, et al. Integrative functional genomics analysis of sustained polyploidy phenotypes in breast cancer cells identifies an oncogenic profile for GINS2. Neoplasia. 2010; $12: 877-88$

16. Ouyang F, Liu J, Xia M, Lin C, Wu X, Ye L, et al. GINS2 is a novel prognostic biomarker and promotes tumor progression in early-stage cervical cancer. Oncol Rep. 2017; 37: 2652-62.

17. Menyhart O, Nagy A, Gyorffy B. Determining consistent prognostic biomarkers of overall survival and vascular invasion in hepatocellular carcinoma. R Soc Open Sci. 2018; 5: 181006

18. Li T, Fan J, Wang B, Traugh N, Chen Q, Liu JS, et al. TIMER: A Web Server for Comprehensive Analysis of Tumor-Infiltrating Immune Cells. Cancer Res. 2017; 77: e108-e10.

19. Liu Y, Yang Y, Luo Y, Wang J, Lu X, Yang Z, et al. Prognostic potential of PRPF3 in hepatocellular carcinoma. Aging (Albany NY). 2020; 12: 912-30.

20. Tu L, Guan R, Yang H, Zhou Y, Hong W, Ma L, et al. Assessment of the expression of the immune checkpoint molecules PD-1, CTLA4, TIM-3 and LAG-3 across different cancers in relation to treatment response, tumor-infiltrating immune cells and survival. Int J Cancer. 2020; 147: 423-39.

21. Pan JH, Zhou H, Cooper L, Huang JL, Zhu SB, Zhao XX, et al. LAYN Is a Prognostic Biomarker and Correlated With Immune Infiltrates in Gastric and Colon Cancers. Front Immunol. 2019; 10: 6.

22. Tang Z, Li C, Kang B, Gao G, Li C, Zhang Z. GEPIA: a web server for cancer and normal gene expression profiling and interactive analyses. Nucleic Acids Res. 2017; 45: W98-W102.

23. Shirabe K, Motomura T, Muto J, Toshima T, Matono R, Mano Y, et al. Tumor-infiltrating lymphocytes and hepatocellular carcinoma: pathology and clinical management. Int J Clin Oncol. 2010; 15: 552-8.

24. Zhao Y, Ge X, He J, Cheng Y, Wang Z, Wang J, et al. The prognostic value of tumor-infiltrating lymphocytes in colorectal cancer differs by anatomical subsite: a systematic review and meta-analysis. World J Surg Oncol. 2019; 17: 85.

25. Grivennikov SI, Greten FR, Karin M. Immunity, inflammation, and cancer. Cell. 2010; 140: 883-99.

26. Zhang Z, Liu S, Zhang B, Qiao L, Zhang Y, Zhang Y. T Cell Dysfunction and Exhaustion in Cancer. Front Cell Dev Biol. 2020; 8: 17.

27. Facciabene A, Motz GT, Coukos G. T-regulatory cells: key players in tumor immune escape and angiogenesis. Cancer Res. 2012; 72: 2162-71.

28. Yu Q, Pu SY, Wu H, Chen XQ, Jiang JJ, Gu KS, et al. TICRR Contributes to Tumorigenesis Through Accelerating DNA Replication in Cancers. Front Oncol. 2019; 9: 516.
29. Huang L, Chen S, Fan H, Ji D, Chen C, Sheng W. GINS2 promotes EMT in pancreatic cancer via specifically stimulating ERK/MAPK signaling. Cancer Gene Ther. 2021;28:839-49.

30. Liu $X$, Sun L, Zhang S, Zhang S, Li W. GINS2 facilitates epithelial-to-mesenchymal transition in non-small-cell lung cancer through modulating PI3K/Akt and MEK/ERK signaling. J Cell Physiol. 2020;235:7747-56.

31. Shen X, Li N, Li H, Zhang T, Wang F, Li Q. Increased prevalence of regulatory $\mathrm{T}$ cells in the tumor microenvironment and its correlation with TNM stage of hepatocellular carcinoma. J Cancer Res Clin Oncol. 2010; 136: 1745-54.

32. Su C, Jia S, Liu H. Immunolocalization of CD163+ Tumor-Associated Macrophages and Symmetric Proliferation of Ki-67 as Biomarkers to Differentiate New Different Grades of Laryngeal Dysplasia. Am J Clin Pathol. 2017; 149: 8-16.

33. Sammarco G, Gadaleta CD, Zuccala V, Albayrak E, Patruno R, Milella P, et al. Tumor-Associated Macrophages and Mast Cells Positive to Tryptase Are Correlated with Angiogenesis in Surgically-Treated Gastric Cancer Patients. Int J Mol Sci. 2018; 19:1176

34. Zhang D, Qiu X, Li J, Zheng S, Li L, Zhao H. TGF-beta secreted by tumor-associated macrophages promotes proliferation and invasion of colorectal cancer via miR-34a-VEGF axis. Cell Cycle. 2018; 17: 2766-78.

35. Song W, Mazzieri R, Yang T, Gobe GC. Translational Significance for Tumor Metastasis of Tumor-Associated Macrophages and Epithelial-Mesenchymal Transition. Front Immunol. 2017; 8: 1106. 